\title{
Advances and Applications of EELS in Biology
}

\section{Richard Leapman and Maria Aronova}

National Institute of Biomedical Imaging and Bioengineering, National Institutes of Health, Bethesda, Maryland, United States

Advances in electron energy-loss spectroscopy (EELS) and energy-filtered transmission electron microscopy (EFTEM) now enable the determination of compositional information from inorganic materials at single-atom analytical sensitivity. These developments include efficient detectors, stable spectrometers, and efficient algorithms for acquiring and processing the resulting spectra and images. The same advances have also greatly enhanced our ability to perform EELS and EFTEM on biological systems, but there are challenges in such applications. First, organic compounds in biological materials have a strong tendency to suffer ionization damage, which alters their structure and elemental composition during analysis. Second, it is often necessary to detect weak spectral features, either due to low concentrations of an element of interest, or because of subtle variations in the spectrum due to changes in molecular composition $[1,2]$.

Information about the composition of biological specimens can be found throughout the electron energyloss spectrum. The low-loss spectrum $(0-20 \mathrm{eV})$ contains fine structures arising from excitations of molecular energy levels, which can be used to estimate the relative amounts of water, proteins, nucleic acids, sugars, and other components in frozen hydrated preparations. Signals from core edges provide quantitative information about a range of endogenous elements within biological structures. For example, phosphorus ( $\mathrm{L}_{2,3}$ edge at $132 \mathrm{eV}$ ) reveals phosphate groups in nucleic acids or attached to phosphorylated proteins. Sulfur ( $\mathrm{L}_{2,3}$ edge at $165 \mathrm{eV}$ ) indicates the presence of proteins that are rich in cysteine or methionine residues, or sulfate groups attached to carbohydrates. The ratios of the major constituents of organic molecules $(\mathrm{C}, \mathrm{N}$, and $\mathrm{O})$ can be quantified by analyzing their $\mathrm{K}$ edges at $285 \mathrm{eV}, 532 \mathrm{eV}$, and 400 $\mathrm{eV}$, respectively, which provides additional compositional information [3]. Certain metal atoms occurring in biological specimens are detectable by sharp resonances (white lines) at their EELS core edges (e.g., the $\mathrm{Ca} \mathrm{L}_{2,3}$ edge at $348 \mathrm{eV}$ ), and the Fe L2,3 edge at $707 \mathrm{eV}$ [4]. Calcium is a particularly important element that is involved in the regulation of numerous cellular processes but its concentration is typically only a few millimoles per kg dry weight, corresponding to just tens of atomic parts per million.

Cells generally contain many types of organelles, and structures of interest tend to be sparsely distributed. It is therefore sometimes necessary to obtain compositional images over relatively wide areas, covering entire sections through cells that have dimensions of $\sim 10 \mu \mathrm{m}$. Such measurements are therefore often best performed in the EFTEM mode. In other applications, it can be essential to employ the high efficiency of the scanning transmission electron microscopy (STEM) mode, whereby a focused probe is scanned across the specimen, and multichannel EELS spectra are collected at each pixel in a hyperspectral image. Use of the STEM-EELS mode is essential when radiation damage is a limiting factor, e.g., imaging chemical composition. Similarly, for the detection of trace elemental concentrations, e.g., calcium, the STEM-EELS acquisition mode is also essential.

Fig. 1 compares the microscope configurations for the EFTEM and STEM-EELS modes and shows how the three-dimensional spectrum-image data are accumulated. The lower part of Fig. 1 lists some potential applications of this type of flexible instrumentation, in both the EFTEM and STEM-EELS modes. 
Adams et al. [5] have developed multicolor electron microscopy using genetically encoded photoactivated singlet oxygen generators for each labeled protein, which polymerize diaminobenzidine introduced into the specimen and which is chelated with a specific rare-earth for each protein. This approach is analogous to the use of genetically encoded co-expressed green fluorescent protein (GFP) in optical microscopy, developed by R.Y. Tsien et al., which are used as probes in optical microscopy. We have also demonstrated that it is possible to use EFTEM imaging in combination with electron tomography to determine the 3D distribution of specific elements such as phosphorus and nitrogen [6]. A challenge of EFTEM imaging at the high-energy edges of the rare-earth $\mathrm{M}_{4,5}$ edges ( $800 \mathrm{eV}$ to $\left.1,400 \mathrm{eV}\right)$ is the weakness of the signal. Direct electron detectors with single electron sensitivity are likely to be necessary for such applications, and are now available for the new generation of Gatan Quantum GIF energy filters [7].

For applications involving detection of near-trace levels of calcium, STEM-EELS imaging provides higher sensitivity than EFTEM. Here, recent advances in EELS performance offer strong advantages. For example, the Gatan Quantum Dual EELS system enables as many as 1,000 parallel spectra to be read out per second [8]. This allows larger areas of a sample to be imaged in a reasonable time, an important factor in the context of biological specimens, where regions of interest can be sparsely distributed, such as synapses in brain. This spectrum-imaging capability is also expected to offer higher sensitivity for imaging trace levels of both endogenous elements such as $\mathrm{Ca}$, and exogenous elements such as manganese used as contrast agents in magnetic resonance imaging. It has already been demonstrated that near-single atom sensitivity is achievable by using STEM-EELS spectrum imaging of biological specimens [9]. In addition, the improved capabilities of STEM-EELS also enable acquisition of low-loss spectrum-images containing large numbers of pixels in the low dose regime [10]. This is likely to increase the range of applications of this technique, including the mapping of water in frozen hydrated cryosections, imaging the distributions of compounds localized in specific organelles, as well as imaging functionalized bionanoparticles in cells. 
EFTEM

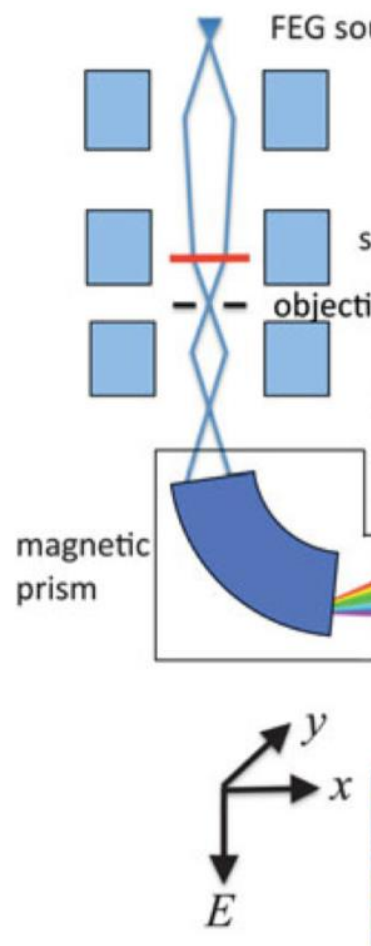

specimen

specimen

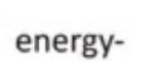

selecting

slit

detector
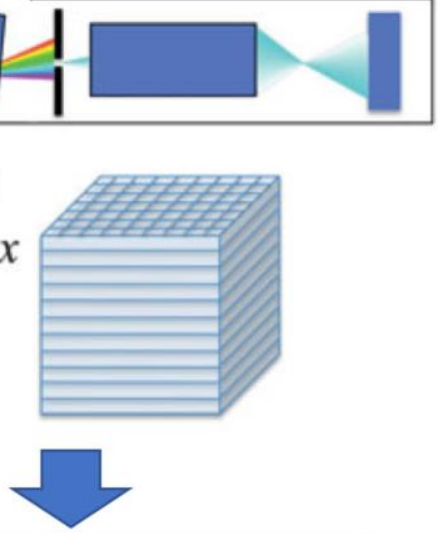

- Multicolor electron microscopy using rare-earth probes to image specific labeled proteins [5]

- Core-edge EFTEM tomography for 3D elemental mapping [6]

- Mapping weak core edges with direct electron detectors ? [7]

\section{STEM-EELS}
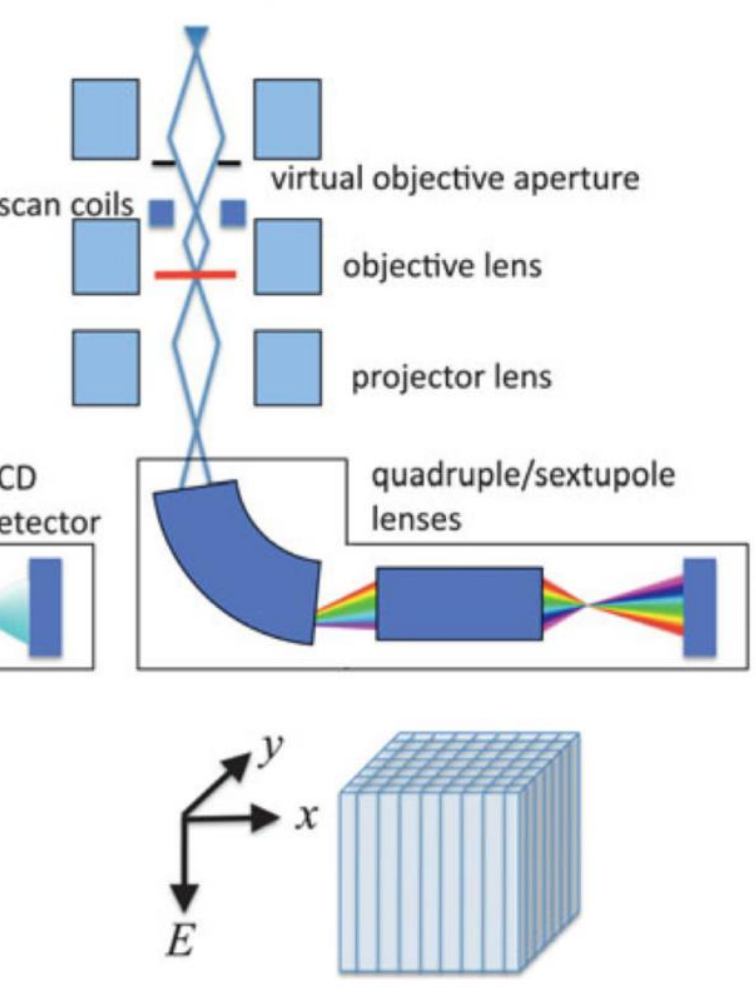

- Spectrum-imaging w/Gatan Dual-EELS

$\mathrm{w} / \sim 1 \mathrm{~ms}$ read-out per pixel and

'simultaneous' low-loss and core-loss [8]

- Near single-atom detection /

trace elemental concentrations [9]

- Mapping biological compounds

w/ low-loss EELS? [10]

Figure 1. Comparison of techniques for compositional imaging in a TEM capable of operating in the EFTEM and STEM-EELS modes. The instrument shown is equipped with a post-column imaging filter although an in-column filter can also be used. The spectrum-images data cubes are indicated for both EFTEM and STEM-EELS, along with some recent capabilities of the techniques. 


\section{References}

[1] RD Leapman, Ultramicroscopy 180 (2017) p. 180.

[2] MA Aronova, RD Leapman, MRS Bull. 37 (2012) p. 53.

[3] RD Leapman, RL Ornberg, Ultramicroscopy 24 (1988) p. 251.

[4] RD Leapman, J. Microsc. 210 (2003) p. 5.

[5] SR Adams et al, Cell Chem. Biol. 23 (2016) p. 1417.

[6] MA Aronova et al, J. Struct. Biol. 161 (2008) p. 322.

[7] JL Hart et al, Sci. Rep. 7(8243) (2017) p. 1.

[8] A Gubbens et al, Ultramicroscopy 110 (2010) p. 962.

[9] RD Leapman et al, Ultramicroscopy 49 (1993) p. 225.

[10] RD Leapman et al, J. Microsc. 177 (1995) p. 18. 